\title{
Limits in the application of harmonic analysis to pulsating stars
}

\author{
J. Pascual-Granado ${ }^{1}$, R. Garrido ${ }^{1}$, and J. C. Suárez ${ }^{1,2}$ \\ 1 Instituto de Astrofísica de Andalucía (CSIC), 18008 Granada, Spain \\ e-mail: [javier;garrido; jcsuarez]@ugr.es \\ 2 Dept. Física Teórica y del Cosmos. Universidad de Granada, 18071 Granada, Spain
}

Received 29 December 2014 / Accepted 4 July 2015

\begin{abstract}
Using ultra-precise data from space instrumentation, we found that the underlying functions of stellar light curves from some AF pulsating stars are non-analytic, and consequently their Fourier expansion is not guaranteed. This result demonstrates that periodograms do not provide a mathematically consistent estimator of the frequency content for this type of variable stars. More importantly, this constitutes the first counterexample against the current paradigm, which considers that any physical process is described by a continuous (band-limited) function that is infinitely differentiable.
\end{abstract}

Key words. asteroseismology - methods: data analysis - stars: oscillations

\section{Introduction}

The unexpected huge number of frequencies found in multiperiodic stars (Poretti 2009) and the ubiquitous presence of correlated noise in the residuals of the fitting of the light curves of pulsating stars could have a common origin.

The necessary condition for a correct Fourier analysis, that is, to have a Fourier expansion, is guaranteed when the function is analytic, otherwise Fourier analysis is not a consistent approximation. In this work we examine the differentiability of the function describing photometric data of pulsating stars obtained by CoRoT (Auvergne et al. 2009) and Kepler (Gilliland et al. 2010). We determine how smoothly the data points of the light curve can be fitted to fully reproduce the function. We call this fine-structure property the connectivity of the function at a given point. We studied this property by means of two numerical approaches: cubic splines - analytic ${ }^{1}$, and autoregressive moving average (Box 1976) methods - non-analytic.

\section{Analyticity}

Fourier analysis is a useful technique for frequency detection. To apply Fourier techniques to a given function, Parseval's theorem (Parseval 1992) must hold. This theorem states that the integral of the squared modulus of a function is equal to the integral of the squared modulus of its Fourier transform. For this to be true, the function must be square integrable. The Fourier expansion of the function converges pointwise almost everywhere when it is square integrable (Carleson 1966). Unlike the Fourier series,

\footnotetext{
1 By definition, only the first and second derivatives are continuous for cubic splines, but we are interested here only in the first derivative. In any case, if the first derivative is not continuous, the functions are non-analytic.
}

which is an infinite sum, the discrete Fourier transform (DFT) and its inverse, the IDFT, which is the analogue to the Fourier series, always converge if the sequence is finite, because they are finite sums. But when the Fourier series of the function is divergent, or not convergent to the value of the function at this point, the DFT no longer provides a mathematical description based on the frequency components of the function. That is, a frequency is only a well-defined physical variable when the Fourier series converges. Therefore, before a Fourier analysis can be applied to a time series, the convergence of the Fourier expansion of the function must be demonstrated.

On the other hand, the analyticity of a function is a sufficient condition for the square integrability (Dijk 2009). A function is said to be analytic if it is infinitely differentiable so that its Taylor expansion is convergent. If this condition is not met, the convergence of the Fourier series is not guaranteed. That is, analyticity implies that Dirichlet conditions for convergence are fulfilled.

In asteroseismogy studies (and often in astrophysics) such a convergence of the Fourier series is assumed, which means that the analyticity of the underlying function is likewise assumed. To date, no methods to verify it have been proposed. Here we propose a practical method to verify the analyticity condition of the underlying function from the observed sampled data, which in consequence provide evidence of the convergence of the Fourier series.

In this sense, analyticity refers in this paper to the property that a given function can be expanded in Fourier series. To study the analyticity of a function, we introduce a numerical approximation for the differentiability of discrete time series: the connectivity ${ }^{2}$.

2 Avoid confusion with graph theory term connectivity (Diestel 2005). 


\section{Connectivity}

Time-dependent astronomical data are given as a discrete sequence of samples from an underlying function, which is a representation of a given physical observable. This latter is understood as the physical quantity that satisfies all the properties $^{3}$ derived from the Lebesgue measure definition (Jech 1997).

The discrete sampled sequence of photometric variations of luminosity and/or radial velocity measurements of pulsating stars is commonly called light curve (or RV). We here consider such a series as a finitely close sequence of data points that samples a function composed of a set of infinitely close data points. Under these conditions, we wish to analyse whether such a light curve fully and unambiguously determines the properties of the sampled function. This is the well-known problem of sampling. It is known (Nyquist-Shannon theorem, Shannon 1949) that this is possible when the sampled function is bandlimited to less than half the sampling rate, that is, the Nyquist frequency. When this happens, the complete information of the continuum (i.e. the sampled function) is contained in the discrete sequence. This implies that all the properties of the function can be studied, even those concerning the point-wise limits of the function. Consequently, we are allowed to adequately evaluate the differentiability of the sampled function.

We now generalize our discrete sequence of data to sample a function composed of a stochastic sequence plus a deterministic contribution that can or cannot have a wave-like structure. Such decomposition is guaranteed under the hypothesis of stationarity by the Wold theorem (Wold 1938). The random component might complicate the differentiability analysis of a time series, but the Kolmogorov continuity theorem allows us to fully characterise the properties of the function (Revuz 1999) in a similar way as is done for pure deterministic signals. This necessarily requires the topological separability of the function, which is applicable under certain technical constraints related with the moments of the data differences, particularly variations at most as those given by a power law (see Appendix A for a discussion on these constraints). When these restrictions are fulfilled, the Kolmogorov theorem states that a stochastic process has a continuous representation.

This is a necessary and sufficient condition for the connectivity analysis that we define in the next section.

\subsection{Definition}

Given a data point from a discrete sequence, we can calculate a forward and backward extrapolation from the subsets bracketing the selected data point. Then, it is possible to check whether both extrapolations converge to the same value and coincide with the value of the selected data point.

The above theoretical framework permits the following selfconsistent definition of the connectivity $C_{n}$ of a data point $y_{n}$ of a discrete sequence that samples the function $F(t)$

$C_{n}=\epsilon_{n}^{f}-\epsilon_{n}^{b}$,

where $\epsilon_{n}^{f}, \epsilon_{n}^{b}$, are the deviations of the forward and backward extrapolations from the sample $y_{n}$

$\epsilon_{n}^{f}=y_{n}^{f}-y_{n}$

$\epsilon_{n}^{b}=y_{n}^{b}-y_{n}$,

\footnotetext{
3 We stress here that an observable of a given physical property is not necessarily coincident with the function that represents it.
}

represented by $y_{n}^{f}$ and $y_{n}^{b}$, respectively. In fact, a discrete approximation of the derivability condition of $\mathrm{F}$ at the point $t_{n}$ can be

$$
\frac{y_{n}^{b}-y_{n+1}}{\Delta t}=\frac{y_{n}^{f}-y_{n-1}}{\Delta t},
$$

where $\Delta t$ is the sampling rate of the sequence. We note that connectivity is closely related to derivability. In fact, we can define a new quantity $\mathcal{D}_{n}$ as

$\mathcal{D}_{n}=\frac{\xi_{n}+y_{n+1}-y_{n-1}}{2 \Delta t}$

which reduces to the typical point derivative (Burden 2011) for discrete data when $\xi_{n}$, the non-differentiable component of the sequence, is zero. Likewise, the connectivity as defined above is a function of $\xi_{n}$, that is

$C_{n}=C\left(\xi_{n}\right)+\epsilon_{n}$

From this equation, when connectivities are not zero but an independent normal stochastic sequence ${ }^{4}$, they can be identified with the random term $\epsilon_{n}$, which is a numerical error. In this case, the non-differentiable component is zero $\left(\xi_{n}=0\right)$, and therefore the derivatives are still well defined. When connectivities are correlated with the signal, the non-differentiable component is different from zero $\left(\xi_{n} \neq 0\right)$, and therefore a derivative cannot be defined. In this case, the function is non-analytic and it does not satisfy the conditions under which Parseval's theorem is demonstrated, meaning that Fourier analysis is not consistent.

In summary, sampled data are obtained through the sampling of functions that need not necessarily be differentiable a priori. Connectivities can be interpreted as jumps in the derivatives giving rise to discontinuities. This makes connectivities mathematically self-consistent. They can be considered as equivalent to the non-differentiability coefficient introduced by Wiener (1923) in a different physical context and allow to check the differentiability of the underlying functions.

\subsection{Method}

The forward and backward extrapolations necessary to obtain the connectivities have to be calculated numerically. Two conceptually different numerical approaches were considered. A comparison of both approaches allows us to confirm the results without ambiguity.

\subsubsection{Cubic splines}

The Stone-Weierstrass theorem (Royden 1988) states that if a function is uniformly continuous in a closed interval, it can be approximated arbitrarily well, that is, as closely as desired, by a polynomial of degree $n, n$ being a natural number. Then, the entire function can be represented by a piecewise polynomial function of finite degree. But piecewise polynomial functions have a drawback, the Runge phenomenon, showing oscillations at the edges of the approximated function. This is equivalent to the Gibbs phenomenon in the Fourier approximation.

Taking all of this into account, we selected piecewise smooth polynomials for our modelling.

Formulated in this general way, any particular property can be extended to any analytic function. That is, if a continuous function can be fitted arbitrarily well with a given cubic spline

\footnotetext{
4 This is the theoretically expected distribution of independent samples. It is usually understood as Gaussian white noise.
} 
parametric model, then the analyticity condition is automatically satisfied, otherwise the convergence of the Fourier series is not guaranteed for that particular function. In short, the cubic spline approach gives a key information about local features of the function that generates the observed discrete sequence.

To calculate the coefficients of the cubic spline functions, a tridiagonal linear system is solved (Boor 1978). The only parameter affecting the spline interpolation that can be adjusted is the number of datapoints of the fitted segments.

\subsubsection{ARMA}

These models (Box 1976) give a parametric representation of a time series as a non-closed recursive formula. Autoregressive modelling has been used before for time-series analysis in asteroseismology (Kovacs 1983), although it has not become popular due to the difficulties for the physical interpretation of the fits, in contrast to the well-known Fourier frequencies.

We are interested here in the presence of any non-analytic content of the underlying function describing the observed signals. In contrast to the cubic splines, ARMA models are able to represent non-analytic functions, thereby allowing us to identify non-analytic signals from the residuals.

The algorithm used to calculate ARMA models is similar to gap-filling algorithm MIARMA (Pascual-Granado 2015). This is based on minimising rms residuals and hence is insensitive to the arrow of time (Scargle 1990). However, here we extrapolate only one point each time, and the segments of the modelled data are short enough to be able to estimate derivatives without bias. The invariance to the arrow of time allowed us to calculate backward extrapolations simply as an inverted forward extrapolation.

Like cubic spline modelling, the accuracy of the extrapolations with ARMA models depends on the number of data points of the segment to be modelled. In addition, ARMA extrapolations depend on two parameters: the orders $p$ and $q$, which are the number of autoregressive coefficients and the number of moving average coefficients, respectively. Hereafter, ARMA descriptions of the time series are denoted by their coefficients as $\operatorname{ARMA}(p, q)$. In general, an ARMA model is able to reproduce a sinus wave with only two terms. Accordingly, the number of terms increases with the frequency content of the signal. The ARMA modelling requires an iterative process involving the following steps:

1. Identification of the order of the starting model using the Akaike criterion (Akaike 1974) that is described in detail in Pascual-Granado (2015). This principally affects the velocity of convergence, but does not modify the final result.

2. Calculation of the parameters using a Steiglitz-McBride algorithm (Steiglitz 1965) given the orders for the AR and MA contributions.

3. Evaluation of the validity of the model based on the goodness of the fit in backward and forward extrapolations. The algorithm starts again with increased orders until a minimum in the residuals is reached.

\section{Connectivity analysis applied to stellar light curves}

The test was applied to the light curves of two delta Scuti stars observed by CoRoT and Kepler satellites: HD 174936 and KIC 006187665. The parameters required for this modelling are specific for each of the studied time series. However, to avoid a misleading interpretation of spurious numerical effects, we maintain the same parameters when characterising the connectivities for both time series. The $\delta$ Scuti star HD 174936 was taken as a reference.

For the first step of the ARMA approach, the initial tentative orders were obtained using autoregressive methods (AR). Then we iterated until minimal randomly distributed residuals were obtained, fixing as optimal orders $p=20$ and $q=1$. Cubic splines and ARMA $(20,1)$ connectivities were then calculated for every point in a segment of 1000 samples of the time series using subsets of 80 data points (40 forward and 40 backward extrapolations). We adopted this number of samples per segment as a balance between computer-time consuming and reliability of the ARMA modelling. We checked that the statistical properties of both approximations do not change significantly for a higher number of data points modelled.

To avoid artifacts we selected data segments with no gaps and homogeneous sampling. In addition, the studied segments were statistically normalised, allowing comparison of the connectivity results obtained using different time series.

\section{1. $H D 174936$}

We first applied the method to the light curve of the multiperiodic $\delta$ Scuti star HD 174936 using as analytic solution the 422 independent frequencies found in its periodogram (García Hernández 2009).

These are distributed in a range of frequencies below $900 \mu \mathrm{Hz}$. This very wide range of excited frequencies is not predicted by any non-adiabatic model describing opacity-driven pulsators.

The numerical methods described above were applied to an analytic model of this star. The time series of this model was calculated using the amplitudes and phases of the frequencies found in García Hernández (2009) for the signal component, and a noise component based on additive Gaussian white noise with a level obtained from the same reference.

For both the observed data (Fig. 1) and the time series of the analytic model (Fig. 2), the connectivities calculated using cubic splines have a higher dispersion than the ARMA ones. Nevertheless, a striking effect is seen in the observed data that is lacking in the analytic model: spline connectivities are not randomly distributed, but are strongly correlated with the signal, suggesting that these data are not sufficiently well described by the analytic model. The same effect is present, although five times smaller in amplitude, for the ARMA results.

The difference between the analytic model and observed data has usually been interpreted as correlated noise caused by the effects of the turbulence present in the envelopes of stars (Chaplin 1997). To check whether the convection is at the origin of this phenomenon, some additional tests were performed by adding non-white noise to the analytic model following the procedure described in Kallinger (2010). A power law with two components (36 ppm and $19 \mathrm{ppm}$ of amplitudes) was added in the frequency domain to the analytic model (see Appendix B). The results of this test do not significantly change the previous ones, that is, correlated connectivities were found. We conclude that the fully developed turbulence as represented by Harvey models (Harvey 1985) is not at the origin of this phenomenon.

\subsection{KIC 006187665}

To discard possible instrumental effects specifically linked to a given instrument, a similar test was applied to the time series 

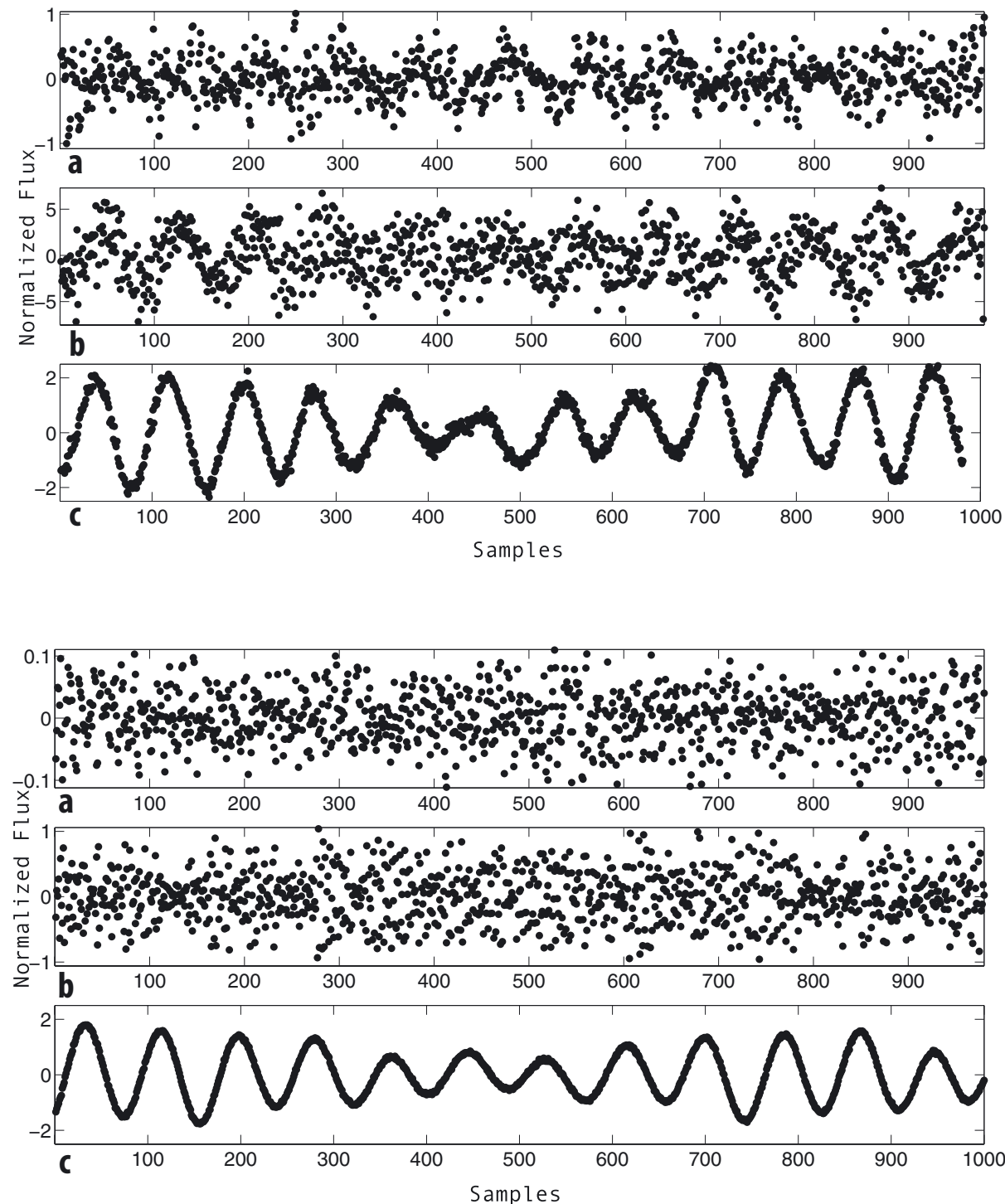

Fig. 1. Connectivities of the CoRoT data for the $\delta$ Scuti star HD 174936. a) ARMA connectivities; b) cubic spline connectivities; c) the original light curve. (Note the different scaling factors in the top and middle panels.)

Fig. 2. Connectivities of an analytic model of the star HD 174936 based on the first 422 frequencies detected using standard Fourier techniques. a) ARMA connectivities; b) cubic spline connectivities; c) the original light curve. (Note the different scaling factors in the top and middle panels.)

from another star, KIC 006187665, observed by the Kepler satellite, and classified as a hybrid Gdor/Dscuti pulsator by Uytterhoeven (2011). The standard Fourier analysis of the light curve supplied by the satellite at a sampling rate of $60 \mathrm{~s}$ (short-cadence regime) yielded 659 significant peaks.

The connectivities corresponding to the Kepler target show a very similar behaviour to those of CoRoT (Figs. 3a,b), that is, cubic spline connectivities show a more dispersed distribution than the ARMA connectivities and are strongly correlated with the original Kepler time series. We calculated the Pearson correlation coefficients for both splines and ARMA connectivities of the two time series studied here, which confirms these results (see Table C.1).

The non-random structures of the connectivities reflect the non-analytic component of the studied function. To characterise them, we calculated their amplitude spectra (Fig. C.1) after verifying its applicability by using the test described in this paper (see Appendix D). The low-frequency range in the case of spline connectivities is very similar to that found in the amplitude spectrum of the original time series. However, a regular pattern can be seen along the frequency axis, increasing in amplitude with frequencies. The ARMA connectivities show some pattern at low frequencies, whereas an almost flat spectrum, appearing as a white-noise spectrum, is obtained at higher frequencies. The amplitudes of the ARMA connectivities are one to two orders of magnitude lower than those calculated with cubic splines.

\section{Discussion and conclusions}

The test introduced here through the connectivities allows us to fully characterise the differentiability of the underlying function of a time series. We remark that although the underlying function can be non-analytic due to the noise itself being non-analytic, our test assumes the non-analyticity of this component and evaluates the deterministic component through the correlation of the connectivities.

We have shown that at least for the stars studied here the functions underlying the observed time series are non-analytic. This comes from the finding that connectivities theoretically expected to be zero or a white-noise distribution are strongly correlated with the signal. We showed this to be an intrinsic property of these functions, which implies that their Fourier expansion convergence is not guaranteed. In addition, the fact that periodograms actually converge to a real value does not guarantee 


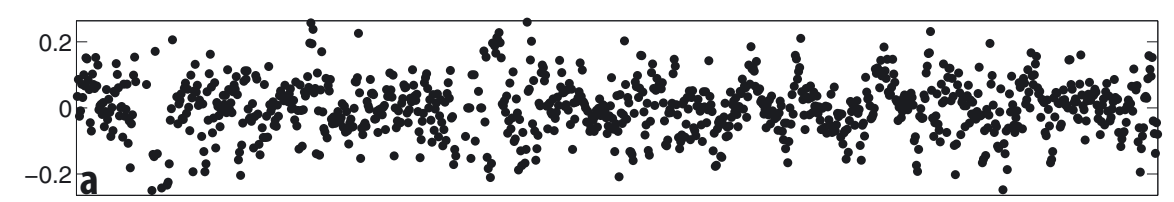

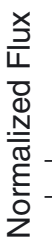

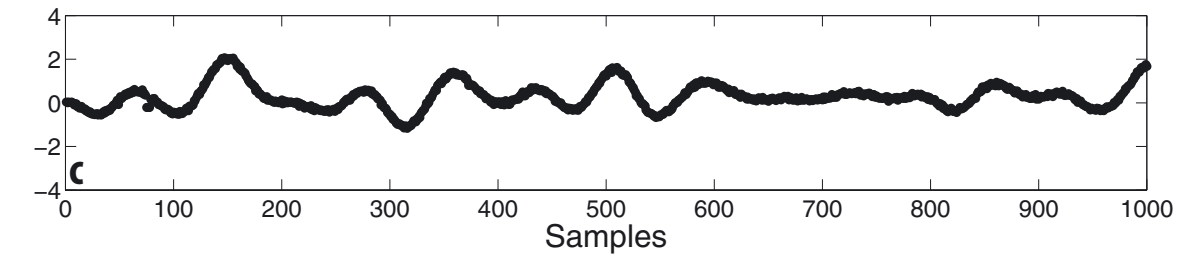

Fig. 3. Connectivities of the Kepler data for the hybrid star KIC 006187665 and the original light curve for comparison. a) ARMA connectivities. b) Cubic spline connectivities. c) Kepler light curve for comparison. (Note the different scaling factors in the top and middle panels.) that this value is an asymptotically $(N \rightarrow \infty)$ unbiased estimator of the spectral density of the underlying function. Therefore, the value estimated for the spectral density at a given frequency bin is not necessarily the true value.

To illustrate the possible relationship between the nonanalyticity and the unexpectedly huge number of frequencies found in multi-periodic stars, we performed a connectivity and frequency analysis of simulated data from a known function, the Weierstrass function (Bailey 2007). This function is originally defined by an infinite sum of harmonic components (see Appendix E), but can be represented using Eq. (E.2) with just a few components (Weisstein 2015). However, it is considered a pathological function since it is continuous everywhere, but differentiable nowhere. We showed that when performing frequency analysis of the simulated data, the residuals of the pre-whitening process do not converge to white noise. We note that very many frequencies are obtained compared with the few components used to generate the function (see Fig. E.3). This is an example of an inconsistency in the application of harmonic analysis that can be related to the non-analyticity of the underlying function.

We do not claim that the Weiertrass function is at the basis of the inconsistencies found in the time series of the two $\delta$ Scuti stars observed from space with different instrumentation, we only used its pathological properties to try to "simulate" the real cases here studied. Although this peculiar function suggests a Fourier expansion in its standard form (Eq. (E.1)), Fourier frequencies, given by an integer number of the argument, cannot be defined. This could point towards a reinterpretation of the term frequency in the context of the harmonic analysis of pulsating stars. The consequences of this new description of frequency are obviously beyond the scope of this paper. Although the origin of these inconsistencies is unclear, our next step is to try to numerically correct the time series to overcome the analyticity test and therefore obtain a cleaner power spectrum.

We conclude that periodograms do not provide a mathematically consistent estimator of the frequency content for stellar variability of the objects here studied. Accordingly, the concept of unambiguous detection of a physical frequency needs to be revised. This constitutes the first counterexample against the current paradigm, which considers that any physical process is described by a continuous (band-limited) function that is smooth and infinitely differentiable.
This could be on the basis of the unknown nature of many phenomena related to the power spectrum of AF pulsating stars: the unexpectedly huge number of frequencies found in multiperiodic stars and the range of these frequencies as mentioned before, and also the ubiquitous presence of correlated noise in the residuals of the fitting of the light curves.

This paper shows an inconsistency in the application of harmonic analysis to some pulsating stars observed by space missions like CoRoT or Kepler. This inconsistency is related with the non-analyticity of the underlying function, the origin of which is still to be unveiled.

Acknowledgements. The CoRoT space mission, launched on December 27, 2006, has been developed and is operated by CNES, with the contribution of Austria, Belgium, Brazil, ESA (RSSD and Science Programme), Germany and Spain. Funding for the Kepler Discovery mission is provided by NASAs Science Mission Directorate. The authors acknowledge support from MINECO and FEDER funds through the Astronomy and Astrophysics National Plan under number AYA2012-39346-C02-01. J.P-G. acknowledges support from MINECO through the FPI grant number BES-2008-008252. J.C.S. acknowledges support by the European project SpaceInn, with reference 312844 within the european SPACE program FP7-SPACE-2011-1, and also acknowledges funding support from the Spanish "Ministerio de Economía y Competitividad" under the "Ramón y Cajal" subprogram.

\section{Appendix A: Kolmogorov continuity theorem}

As mentioned in Sect. 3, this theorem (Revuz 1999) allow us to fully characterise the properties of a stochastic function through its continuous representation. The theorem comes after the following definition:

Def:: an extension $\left(\tilde{Y}_{t}\right)$ of an stochastic process $\left(Y_{t}\right)$ is a process such that for every $t \geq 0, P\left(Y_{t}=\tilde{Y}_{t}\right)=1$. As a corollary, if the process $\left(Y_{t}\right)$ satisfies for all times $T>0$ and $0 \leq s, t \leq T$,

$E\left(\left\|Y_{t}-Y_{s}\right\|^{\alpha}\right) \leq c|t-s|^{1+\varepsilon}$,

with $\alpha, \varepsilon, c$ positive constants, then there exists an extension of the process $\left(Y_{t}\right)$ that is a continuous process whose paths are almost surely continuous. This is known as topological separability.

These conditions are quite mild, and they allow us to consider stochastic processes as continuous paths instead of discrete values, as it is the case for deterministic functions. 
It can be demonstrated that these conditions are fulfilled by any white-noise process and even for a non-Gaussian noise having a spectral density proportional to $f^{\alpha}$ (with $\alpha<0$ ), that is, the so-called coloured noise.

As an example, a real-valued Wiener process (so-called coloured noise proportional to $v^{-2}$ ) has continuous trajectories almost surely, in mathematical jargon, by this theorem. This means that it admits a separable and progressively measurable extension (Capasso 2005).

In this work we refer to the stochastic component of a time series with the properties of Gaussian white noise.

\section{Appendix B: Analytic model construction}

The numerical methods described in the main text were applied to an analytic model of $\delta$ Scuti HD 174936. This model was built using the amplitudes and phases of the first 422 frequencies found using a standard Fourier analysis in García Hernández (2009), fitted to an harmonic Fourier-like expansion of the form

$x^{a}\left(t_{i}\right)=\sum_{i=1}^{n} A_{i} \cos \left(2 \pi v_{j} t_{i}+\phi_{j}\right)+N^{\mathrm{w}}+N^{\mathrm{nw}}$,

where $A_{i}, v_{j}$, and $\phi_{j}$ represent the observed amplitudes, frequencies, and phases, respectively. The $N^{\mathrm{w}}+N^{\mathrm{c}}$ terms represent the white noise, defined as normalised distribution scaled to the data,

$N^{\mathrm{w}}=\left\langle\sigma^{N}\right\rangle$

and the non-white noise, calculated as

$N^{\mathrm{nw}}=\mathcal{F}^{-1}\left[\tilde{N}^{\mathrm{nw}}\right]$, where $\tilde{N}^{\text {nw }}$ is defined in the transformed domain as

$\tilde{N}^{\mathrm{nw}}=\left[\sum_{k=1}^{m} \frac{a_{k}}{1+\left(\frac{v}{b_{k}}\right)^{c_{k}}}\right]^{1 / 2} \mathrm{e}^{\mathrm{i} \theta}$.

where $a_{k}$ and $b_{k}$ are free parameters. The values of $c_{k}=4$ and $m=2$ were adopted following Kallinger \& Matthews (2010). This noise was calculated setting the $\theta$ phases equal to a random uniform distribution in the $[-\pi, \pi]$ interval. The light curve so constructed is assumed to be caused by the oscillation modes of the pulsating star plus granulation noise modelled as a fully developed turbulence regime (Harvey 1985) and given by Eq. (B4).

\section{Appendix C: Pearson correlation coefficients}

The correlations between the connectivities and their corresponding original time series are evident in Fig. C.1, even so, we calculated their corresponding standard Pearson coefficient to quantify the correlation between these two series (see Table C.1). We also calculated $P$-values to test the null hypothesis (i.e. no correlation). $P$-values were calculated using a t-Student distribution. Considering the $P_{\mathrm{s}}$ negligible values obtained for the time series of the selected stars, the null hypothesis must be rejected under any computable limit of validity, that is, the two series are strongly correlated. On the other hand, for the case of ARMA $\left(P_{\mathrm{a}}\right)$, this limit can be set as an standard choice for this parameter, for instance, $P<0.05$, that is, the ARMA connectivities would be correlated with the original signal in only $5 \%$ of the cases.

Since ARMA models can capture the non-analytic component of the signal, this non-correlation of the connectivities is expected.

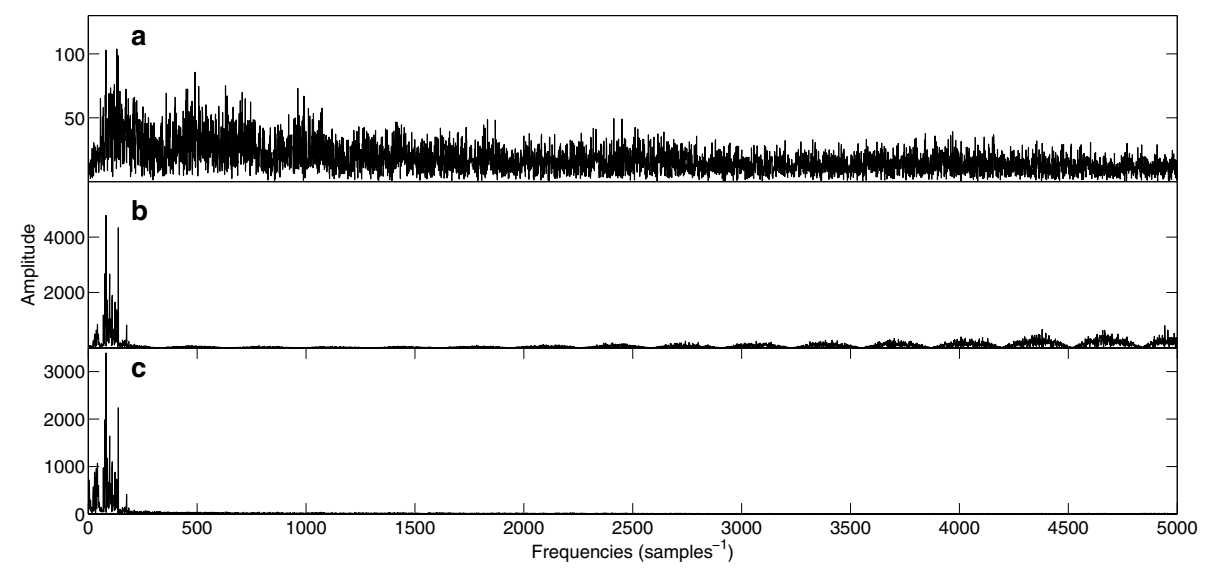

Fig. C.1. Amplitude spectrum of the connectivities of the Kepler data for the hybrid star KIC 006187665. From top to bottom: panel a) shows the amplitude spectrum of the ARMA connectivities, panel b) amplitude spectrum of the cubic spline connectivities, panel c) amplitude spectrum of the original light curve for comparison.

Table C.1. Pearson correlation coefficients $(\rho)$ and the associated probabilities of no-correlation $(P)$ for ARMA and splines connectivities for the time series considered in this work.

\begin{tabular}{ccccc}
\hline \hline Time series & $\rho_{\mathrm{a}}$ & $P_{\mathrm{a}}$ & $\rho_{s}$ & $P_{\mathrm{s}}$ \\
\hline CoRoT 7613 & -0.0075 & 0.8139 & 0.4243 & $5.8247 \mathrm{e}-45$ \\
Analytic model & 0.0072 & 0.8199 & $8.371 \mathrm{e}-4$ & 0.9789 \\
KIC 006187655 & -0.0257 & 0.4176 & 0.5553 & $5.8039 \mathrm{e}-82$ \\
\hline
\end{tabular}

Notes. Subscripts a and s refer to the ARMA and splines approaches, respectively. 


\section{Appendix D: Second-order connectivities}

Analyticity, understood as infinite differentiability, guarantees the convergence of the Fourier expansion of a function, so allowing to perform Fourier analysis. The test described in this work indicates whether the function underlying a time series is differentiable or not. Considering the connectivities as a new time series, the calculation of the connectivities of the connectivities (from now on second-order connectivities) informs us about their differentiability. If, using Pearson correlation coefficients, second-order connectivities are independent, normal, and randomly distributed, then connectivities are differentiable. It is thus legitimate to perform a Fourier analysis of them.

We applied this method presented to the spline connectivities of the stellar light curve of KIC 006187665 (Fig. D.1, panel a). In contrast to the connectivities, second-order connectivities calculated using the spline approximation (Fig. D.1, panel b) show a normal white-noise distribution. These, when calculated with splines, show that the connectivities are differentiable. The ARMA approximation does not provide better fits than the cubic splines in this case.

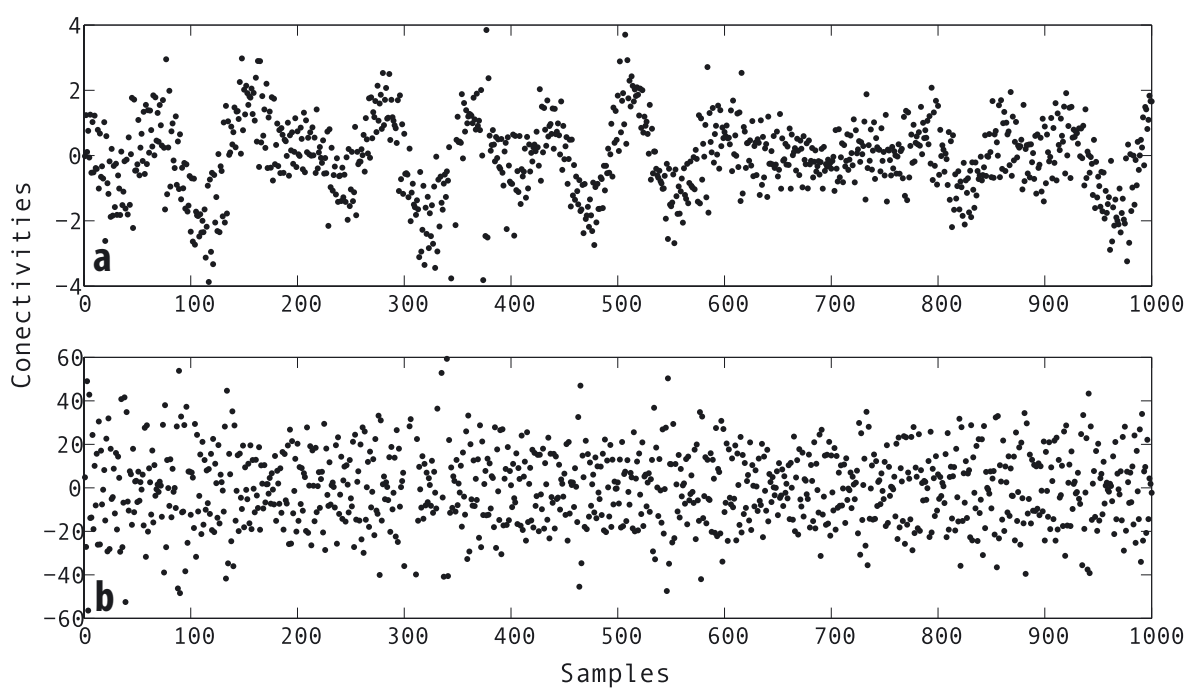

Fig. D.1. Second-order connectivities calculated using the cubic spline approximation for Kepler data of the hybrid star KIC 006187665 and the first-order connectivities for comparison. From top to bottom: panel a) first-order spline connectivities; panel b) second-order spline connectivities. Note that the correlation present in the first is lost in the last. 


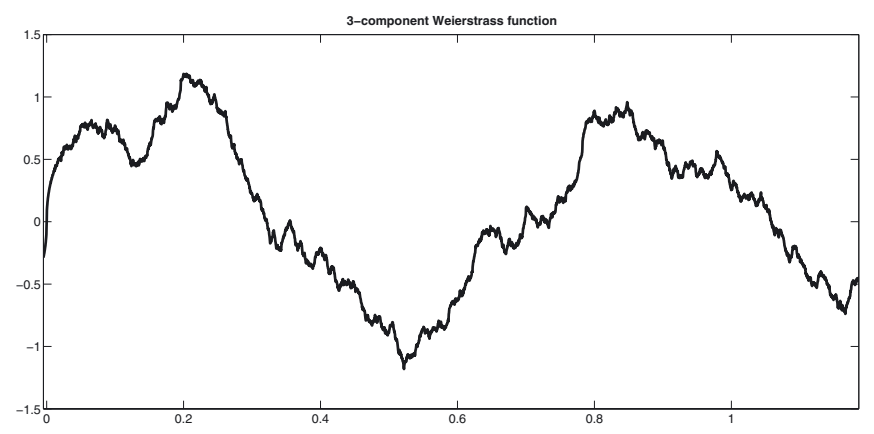

Fig. E.1. Realization of the Weierstrass function with three components corresponding to three different q values as indicated in Eq. (E.2).

\section{Appendix E: Non-analytic model}

Frequency and connectivity analysis has been applied to a non-analytic model based on a numerical realization ${ }^{5}$ of the Weierstrass function. This is an extreme case of a function exhibiting non-analyticity because it is continuous everywhere, but differentiable nowhere.

\section{E.1. Definition}

The original definition of the Weierstrass function is

$W(x)=\sum_{n=0}^{\infty} a^{n} \cos \left(b^{n} \pi x\right) \quad 0<a<1, \quad a \cdot b>1+\frac{3}{2} \pi$,

and $b, a$ positive odd integer number. For rational numbers $x=$ $\frac{p}{q}$, which is our case, $W(x)$ can be calculated with a finite sum such as:

$W\left(\frac{p}{q}\right)=\frac{\pi}{4 q^{2}} \sum_{n=1}^{q-1} \frac{\sin \left(\frac{n^{2} p \pi}{q}\right)}{\sin ^{2}\left(\frac{n \pi}{2 q}\right)}$.
This formula allows us to generate an exact numerical realization of the Weierstrass function without calculating an infinite sum.

For the non-analytic model that we used, we generated three realizations of $W(x)$ with different $q$ values and obtained a time series of 30000 data points by iterating on $p$ and adding each $q$ component.

\section{E.2. Connectivity analysis}

We appied the numerical techniques introduced before to the time series generated as described in the previous section. Connectivities calculated using the spline approximation are clearly correlated (Fig. E.2) and ARMA connectivities are not. These results are consistent with the definition and properties of the Weierstrass function and demonstrate with the analytic model discussed in Appendix B the validity of the connectivity analysis as a test for the analyticity of the underlying function of a time series.

\section{E.3. Frequency analysis}

A power spectrum of the time series of the non-analytic model and frequency detection was performed using SIGSPEC (Reegen 2007) to check the effect of the non-analyticity in a standard Fourier analysis. A sequence of power spectra was obtained during the pre-whitening process of different sets of frequencies (Fig. E.3). The power spectrum at each step was significantly different from a white-noise spectrum, and the pre-whitening process continued until the program stopped at 738 frequencies when the limit in significance was reached.

In conclusion, a frequency detection procedure using standard Fourier analysis is not convergent. This is because in this case the power spectrum calculated with a DFT is not a consistent estimator for the original frequency content as a consequence of the non-analyticity of the underlying function.

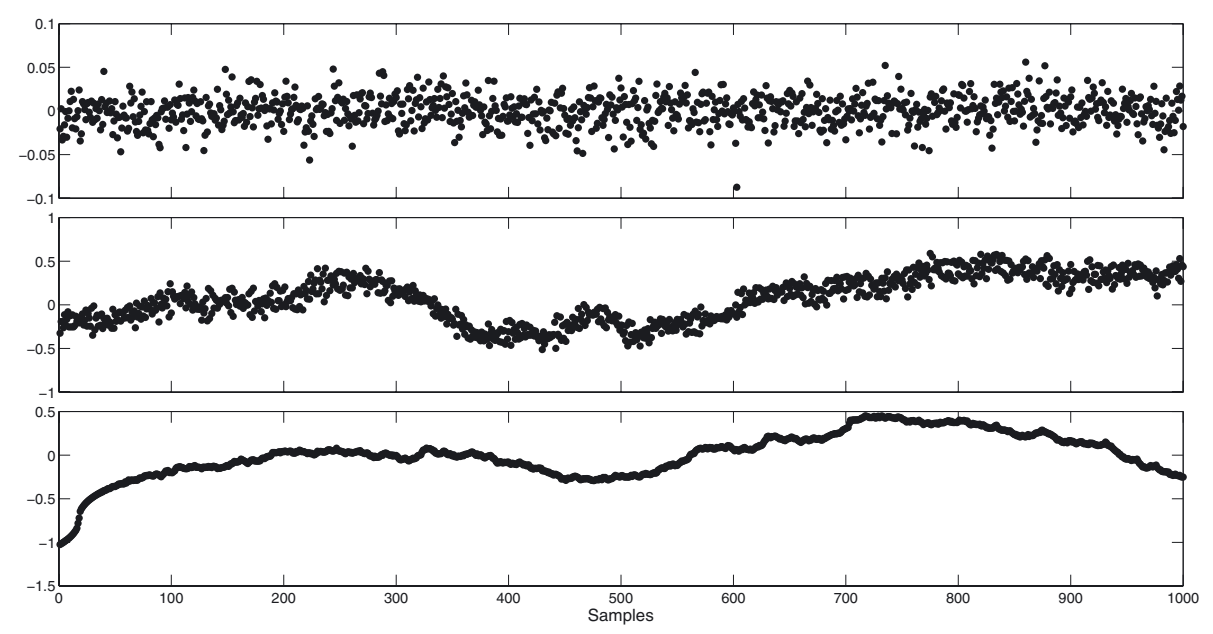

Fig. E.2. Connectivities of a realization of a three-component Weierstrass function. Upper panel: ARMA connectivities. Middle panel: cubic spline connectivities. Lower panel: original light curve. (Note the different scaling factors.)

\footnotetext{
5 Obtained by sampling the continuous function.
} 
J. Pascual-Granado et al.: Limits in the application of harmonic analysis to pulsating stars

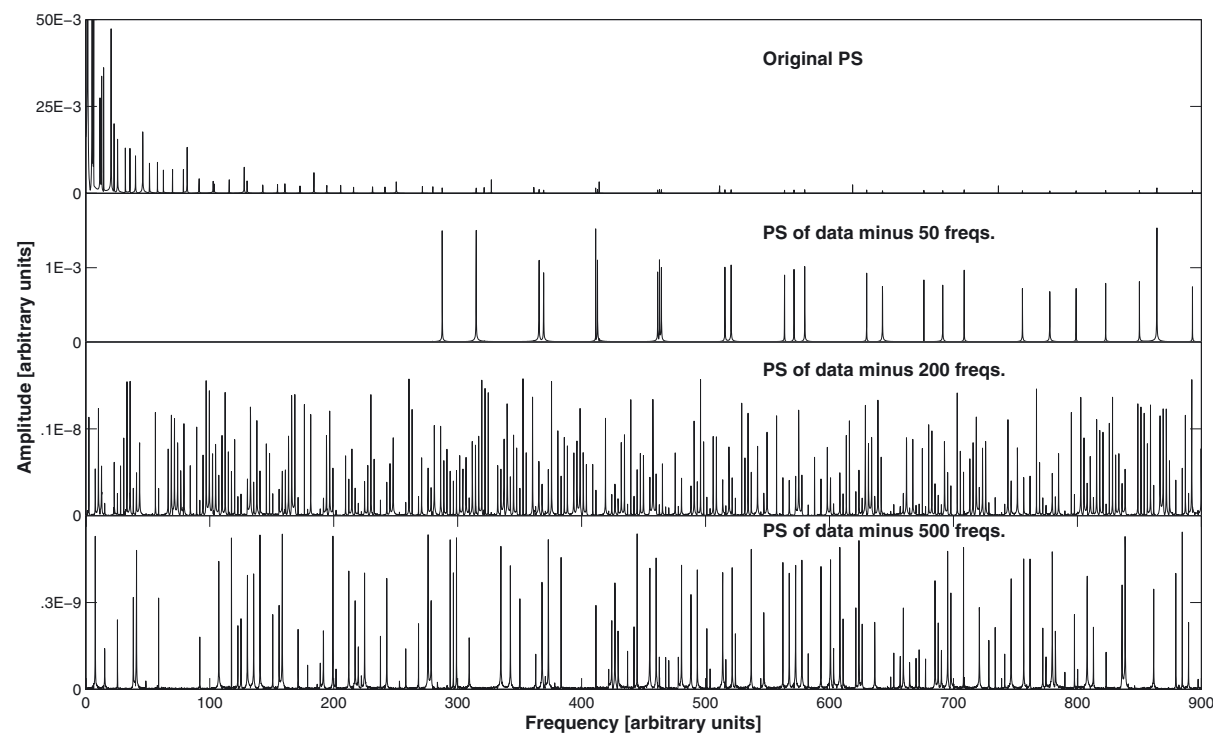

Fig. E.3. Power spectrum of the time series generated with a realization of a threecomponent Weierstrass function (top panel) and the power spectra obtained during different steps in the pre-whitening process (succesive panels).

\section{References}

Akaike, H. 1974, IEEE Trans. Automatic Control, AC-19, 716

Auvergne, M., Bodin, P., Boisnard, L., et al. 2009, A\&A, 506, 411

Bailey, D. H. 2007, Experimental mathematics in action, Ak Peters Series

Boor, Carl de 1978, A Practical Guide to Splines, Series: Applied Mathematical Sciences, 1st edn. (New York Berlin Heidelberg: Springer), 27

Box, George E. P., Jenkins, \& Gwilym 1976, M. Time Series Analysis: Forecasting and Control (San Francisco, CA: Holden-Day)

Burden, R. L., \& Faires, J. D. 2011, Numerical Analysis, 9th edn. (Boston, MA: Brooks/Cole)

Capasso, V., \& Bakstein, D. 2005, An Introduction to Continuous-Time Stochastic Processes, Birkhäuser

Carleson, L. 1966, On convergence and growth of partial sums of Fourier series, Acta Math., 116, 135

Chaplin, W. J., Elsworth, Y., Howe, R., et al. 1997, MNRAS, 287, 51

Diestel, R. 2005, Graph Theory, Electronic, 12

Dijk, G. van 2009, Introduction to harmonic analysis and generalized Gelfand pairs (Berlin, New York: Walter De Gruyter)

García Hernández, A., Moya, A., Michel, E., et al. 2009, A\&A, 506, 79

Gilliland, R. L., Brown, T. M., \& Christensen-Dalsgaard, J. 2010, PASP, 122, 131
Harvey, J. 1985, Proc. ESA Workshop on Future Missions in Solar, Heliospheric \& Space Plasma Physics, Garmisch-Partenkirchen, 199

Jech, T. J. 1997, Set Theory, 2nd edn. (Berlin: Springer-Verlag)

Kallinger, T., \& Matthews, J. M. 2010, ApJ, 711, L35

Kovacs, G. 1983, Sol. Phys., 83, 123

Kaplan, W. 1992, Advanced Calculus, 4th edn. Reading (MA: Addison-Wesley)

Pascual-Granado, J., Garrido, R., \& Suárez, J. C. 2015, A\&A, 575, A78

Poretti, E., Michel, E., Garrido, R., et al. 2009, A\&A, 506, 85

Revuz, D., \& Yor, M. 1999, Continuous Martingales and Brownian Motion, A Series of Comprehensive Studies in Mathematics (Springer), 293

Reegen, P. 2007, A\&A, 467, 1353

Royden, H. L. 1988, Real Analysis, Prentice-Hall

Scargle, J. D. 1990, AJ, 359, 469

Shannon, C. E. 1949, Communication in the presence of noise, Proc. IEEE, 37,

Steiglitz, K., \& McBride, L. E. 1965, IEEE Trans. Automatic Control AC-10, 461

Weisstein, E. W. 2015, Weierstrass Function. From MathWorld-A Wolfram Web Resource http://mathworld.wolfram.com/WeierstrassFunction. html

Wiener, N. 1923, Diff. Space. J. Math. Phys., 2, 131

Wold, H. 1938, A Study in the Analysis of Stationary Time Series 2nd edn., Uppsala. Almqvist and Wiksell

Uytterhoeven, K., Moya, A., Grigahcène, A., et al. 2011, A\&A, 534, A125 